\title{
The measles, mumps, and rubella vaccine was not associated with autism in children
}

\author{
Madsen KM, Hvid A, Vestergaard M, et al. A population-based study of measles, mumps, and rubella vaccination and \\ autism. N Engl J Med 2002;347:1477-82.
}

\author{
QUESTION: In children, is the measles, mumps, and rubella (MMR) vaccine associated \\ with autism?
}

\section{Design}

Population based cohort study with data obtained by database linkage.

\section{Setting}

Denmark.

\section{Participants}

537303 children (51\% boys).

\section{Assessment of risk factors}

MMR vaccination status at 15 months of age was obtained from the Danish National Board of Health (compiled by general practitioner reports). Data on birth weight, sex, gestational age, family socioeconomic status, and mother's education were also collected.

\section{Main outcome measure}

Children's autism status was obtained from the Danish Psychiatric Central Register; diagnoses of autistic disorder or another autistic spectrum disorder were assigned by specialists in child psychiatry. When a child was diagnosed with both autistic disorder and $\geq 1$ other autistic spectrum disorder, the diagnosis was classified as autistic disorder

\section{Main results}

The 537303 children had follow up for a total of 2129864 person years. 440655 children $(82 \%)$ had received the MMR vaccine; mean age at time of vaccination was 17 months. 316 children had autistic disorder (mean age at diagnosis 4 y, 3 mo) and 422 children had other autistic spectrum disorders (mean age at diagnosis 5 y, 3 mo). After adjustment for age, calendar period, sex, birth weight, gestational age, mother's education, and socioeconomic status, no increase in risk of autistic disorder or other autistic spectrum disorders was seen in children who received the MMR vaccine compared with those who did not receive the vaccine (table). The risk of

Relative risk of autistic disorder or other autistic spectrum disorder in children who received the measles, mumps, and rubella $(M M R)$ vaccine v children who were unvaccinated ${ }^{*}$

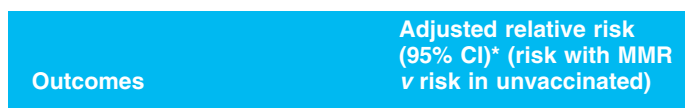

Autistic disorder 0.92 (0.68 to 1.24$)$

\section{Other autistic spectrum disorder 0.83 (0.65 to 1.07)}

*Adjusted for age, calendar period, sex, birth weight, gestational age, mother's education, and socioeconomic status. Both adjusted relative risks are nonsignificant. autistic disorder was not associated with age at time of vaccination $(\mathrm{p}=0.23)$, time interval since vaccination $(\mathrm{p}=0.42)$, or calendar period at time of vaccination $(\mathrm{p}=0.06)$.

\section{Conclusion}

In children, the measles, mumps, and rubella vaccine was not associated with autism.

\section{COMMENTARY}

The possible association between MMR vaccination and autistic spectrum disorders has been controversial since the original report by Wakefield et al ${ }^{1}$ in 1998. In spite of its poor methodological quality, the original study by Wakefield et al has been widely reported in the media resulting in worldwide concern about the safety of the MMR vaccine. Since that time, several studies of good quality have not shown an association between MMR vaccination and autistic spectrum disorders. Several worldwide national and international medical organisations and government organisations and committees have published statements confirming the lack of association between the MMR vaccine and autistic spectrum disorders and have recommended the continued use of the vaccine..-3 Many parents and some professionals remain unconvinced, and there has been a decline in vaccination rates and an increase in morbidity and mortality, particularly from measles. ${ }^{4}$

The study by Madsen et al adds further strong evidence of the lack of association between MMR vaccination and autistic spectrum disorders. This is the highest quality and largest study done to date to address the question of this association. Madsen et al used national databases to study the entire Danish population of children born in an 8 year period. No evidence existed for an association between MMR vaccination and autistic spectrum disorders. The strength of the evidence for the lack of association can be seen in the narrow confidence intervals shown in the study. Physicians who counsel parents and guardians about the MMR vaccine can use the results of this study to further strengthen the argument for the safety of the MMR vaccine. Pamela J Frid, MD University of Western Ontario London, Ontario, Canada

1 Wakefield AJ, Murch SH, Anthony A, et al. Ileal-lymphoidnodular hyperplasia, non-specific colitis, and pervasive 41.

2 Strauss B, Bigham M. Does measles-mumps-rubella (MMR) vaccination cause inflammatory bowel disease and autism? Can Commun Dis Rep 2001:27:65-72.

Global Advisory Committee on Vaccine Safety. Wkly Epidemiol Rec 2003;78:17-20.

Measles, mumps and rubella vaccine (MMR). Department of Health. http://www.doh.gov.uk/mmr/index.html
Sources of funding: Danish National Research Foundation National Vaccine Program Office and National Immunization

Program; Centers for Disease Control and Prevention; and National Alliance for Autism Research.

For correspondence: Dr K M Madsen, Danish Epidemiology Science Center, Aarhus $C$ Denmark. kmm@dadlnet.dk

A modified version of the abstract appears in Evidence-Based Nursing. 